\title{
Temperature dependence of hypersound attenuation in silica films via picosecond acoustics
}

\author{
A. Huynh,${ }^{1, *}$ E. Péronne,${ }^{1}$ C. Gingreau,${ }^{1}$ X. Lafosse,${ }^{2}$ A. Lemaître ${ }^{2}$ B. Perrin,${ }^{1}$ R. Vacher,${ }^{3}$ B. Rufflé, ${ }^{3}$ and M. Foret ${ }^{3}$ \\ ${ }^{1}$ Sorbonne Universités, CNRS-UPMC Univ. Paris 06, UMR 7588, \\ Institut des Nanosciences de Paris, F-75005 Paris, France \\ ${ }^{2}$ Centre de Nanosciences et de Nanotechnologies, CNRS, Univ. Paris-Sud, \\ Université Paris-Saclay, C2N-Marcoussis, 91460 Marcoussis, France \\ ${ }^{3}$ L2C, University of Montpellier, CNRS, Montpellier, France
}

(Dated: 6 décembre 2017)

\begin{abstract}
We report picosecond acoustic measurements of longitudinal sound dispersion and attenuation in an amorphous $\mathrm{SiO}_{2}$ layer at temperatures from $20 \mathrm{~K}$ to $300 \mathrm{~K}$ over frequencies ranging from about $40 \mathrm{GHz}$ to $200 \mathrm{GHz}$. The sample is a radio frequency cathodic sputtered silica layer grown on a sapphire substrate with an aluminum film transducer deposited on top. Acoustic attenuation is evaluated from the simultaneous analysis of three successive echoes using transfer matrix calculation. Results are found to follow rather well a model combining coupling to thermally activated relaxations of structural defects and interactions with thermal vibrations. This leads to a non-trivial variation of the attenuation coefficient with frequency and temperature. The number density of relaxing defects in the $\mathrm{SiO}_{2}$ layer is found to be slightly higher than that in bulk v-SiO 2 . In contrast, similar anharmonic contribution to acoustic absorption is observed in both systems. The velocity variations are also measured and are compared to the dynamical velocity changes deduced from the sound attenuation.
\end{abstract}

PACS numbers: 62.30.+d, 62.40.+i, 63.50.-x, 78.47.jg

\section{INTRODUCTION}

Thin films of amorphous silicon dioxide $\left(\mathrm{SiO}_{2}\right)$ are key materials for a variety of applications that range from insulating layers in integrated circuits to overlays for surface acoustic wave (SAW) devices or supports for metal nano-particles in sensors. The physical properties of the films are often crucial for the performances of the aforementioned applications. It is, however, empirically known that properties of $\mathrm{SiO}_{2}$-films may considerably vary with preparation details. For instance, the quality factor of SAW resonators is observed to vary with deposition temperature of the $\mathrm{SiO}_{2}$ overlay due to excess acoustic loss. ${ }^{1}$ Deposited $\mathrm{SiO}_{2}$ layers are also key materials for some precision experiments in which reduction of thermal noise is of crucial importance. The latter arises in part from internal friction within the material forming the resonator. ${ }^{2}$ For instance, recent work has revealed that losses in the optical coating $\left(\mathrm{SiO}_{2} / \mathrm{Ta}_{2} \mathrm{O}_{5}\right.$ stack $)$ of resonant-mass detectors for gravitational waves have a large contribution to the overall thermal noise. ${ }^{3,4}$ It is therefore of fundamental interest to investigate the dissipative mechanisms at the origin of acoustic losses in amorphous dielectric layers and in particular to compare them to those of the bulk material, including the prototypical vitreous silica $\mathrm{v}-\mathrm{SiO}_{2}$.

Several damping mechanisms have been identified in glasses. A major one is assigned to the interaction of sound with the motion of structural entities, called defects. Thermally activated relaxation (TAR) processes associated to the defects give rise to an absorption peak at ultrasonic frequencies and below. The latter occurs in the interval $30 \mathrm{~K}$ to $70 \mathrm{~K}$ for $\mathrm{v}-\mathrm{SiO}_{2} \cdot{ }^{5-7}$ The frequency de- pendence of the resulting acoustic attenuation $\alpha$ at any given temperature $T$ may be described in terms of the well-known Debye equation:

$$
\alpha(\omega, T) \propto \int G(\tau, T) \frac{\omega^{2} \tau}{1+\omega^{2} \tau^{2}} d \tau
$$

where $G$ describes the distribution of the relaxation times $\tau$ of the defects at $T$. A good description of absorption data is generally obtained assuming thermal activation in asymmetric double-well systems, the asymmetry being associated to quenched spatial disorder. ${ }^{8}$ Regardless of the form of the distribution $G$, Eq. 1 always gives an attenuation whose frequency dependence is slower than quadratically. As in defect-free single crystals, sound damping originating from anharmonic interactions with the thermally excited phonons is also anticipated in glasses ${ }^{9}$ and quasi-crystals ${ }^{10}$. In the Akhiezer treatment ${ }^{11}$, the sound wave perturbs the phonon frequencies and thus the phonon populations of the thermal bath which return to equilibrium within the mean thermal lifetime $\tau_{\text {th }}$ of the dominant vibrations. The relaxation is most efficient for acoustic frequencies $\omega$ for which $\omega \tau_{\text {th }} \sim 1$. As $\tau_{\text {th }}$ is short it ought to contribute to acoustic damping at high $\omega$. An estimate of the relative contribution to sound attenuation in vitreous silica of TAR and Akhiezer mechanism has been provided quite recently in Ref. 12 as part of Brillouin-scattering measurements. Below $10 \mathrm{~K}$ and at still lower $T$, attenuation is produced by interaction of sound with tunneling motions of entities also in asymmetric double-wells. ${ }^{13,14}$ Those tunneling systems give rise to low-energy states which determine the vibrational dynamics of glasses at low $T$.

At extremely high frequency, nearing $\mathrm{THz}$ range, an 


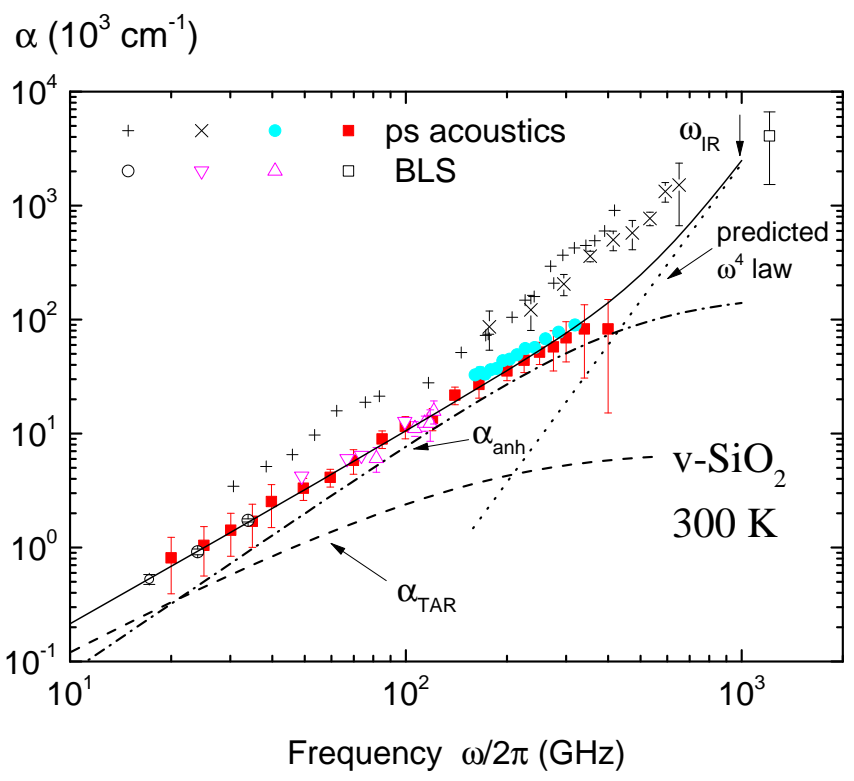

Figure 1. (color online) Collection of energy attenuation coefficient for LA waves in $\mathrm{v}-\mathrm{SiO}_{2}$ at $300 \mathrm{~K}$ deduced from different spectroscopies: visible Brillouin light scattering (BLS) results $^{22}(\mathrm{o})$, ultra-violet BLS ones $\left(\nabla^{23,24} \triangle^{25}\right)$ and ps acoustics results in $\mathrm{SiO}_{2}$ layers by Ayrinhac et al. ${ }^{26}(\bullet)$ and Klieber et al. $^{27}(\mathbf{\square})$ as well as the early data from Zhu et al. ${ }^{28}(+)$ and the high frequency ones from Wen et al. ${ }^{29}(\times)$ which both seem overestimated. The arrow indicates the Ioffe-Regel limit for LA waves from Ref. 17. The point above the Ioffe-Regel frequency $\omega_{\text {IR }}$ is from Brillouin x-ray scattering experiment ${ }^{20}$. The solid line is the estimated sum of TAR (dashed line) and anharmonicity (dash-dot line) contributions plus the predicted $\omega^{4}$ law $^{30}$ (dotted line) as explained in text.

ultimate damping mechanism is inferred from the low$T$ universal plateau in thermal conductivity of glasses. ${ }^{15}$ The latter could arise from Rayleigh type scattering by disorder or resonant coupling to the excess modes manifested by the boson peak. Both mechanisms should give rise to a dramatic increase of $\alpha$ with frequency. A strong damping behavior, $\alpha \propto \omega^{4}$, has been indeed evidenced beyond $\sim 1 \mathrm{THz}$ in few glasses ${ }^{16-19}$ (other than silica) owing to advances in Brillouin scattering of x-rays. ${ }^{20,21}$

Much efforts have been made recently to explore the onset of the $\omega^{4}$ law over the anharmonicity damping in $\mathrm{SiO}_{2}$ layers using the great potential of ultrafast acoustic techniques. ${ }^{26,27,29,31-33}$ However, it could not be observed until now. Fig. 1 illustrates the available attenuation results at hypersonic frequencies which were acquired at room $T$. Data at low frequency obtained by Brillouin light scattering (BLS) in the visible ${ }^{22}$ and ultra-violet ${ }^{23-25}$ regions of the spectrum are shown as opened markers. Following the work of Vacher et al. ${ }^{12}$ one can anticipate the contributions of the above mentioned TAR and anharmonicity mechanisms shown as dashed and dash-dot lines, respectively. Some of the ps acoustics results ${ }^{26,27}$ remarkably fit the expectation from $\left(\alpha_{\mathrm{TAR}}+\alpha_{\mathrm{anh}}\right)$ up to about $300 \mathrm{GHz}$. This suggests that the damping model as detai- led in Ref. 12 is working well even above the BLS frequencies. We also consider in Fig. 1 the data from the historical experiment by Zhu et al. ${ }^{28}$, the very first of its kind, albeit the associated results (like those from Ref. 29) appear now clearly overestimated in light of the overall results. Finally, the dotted line in Fig. 1 is a quantitative prediction from the soft potential mode ${ }^{30}$, the arrow indicating the anticipated Ioffe-Regel limit for LA waves from Ref. 17. The solid line represents the whole damping which is therefore expected at room $T$. One immediate idea to test the overall scenario would be to perform experiments at lower temperatures. On cooling, the TAR contribution is expected to prevail against anharmonicity until sufficiently high frequencies are reached. Then by further increasing frequency, as the $\omega^{4}$ law should be $T$ independent, it should become dominant as $T$ is decreased reducing the anharmonicity damping. However, no significant $T$-dependence of phonon attenuation was found over the range $30-440 \mathrm{GHz}$ in the seminal work by Maris and co-workers. ${ }^{28}$

In this work, we re-investigate sound dispersion and attenuation in a $\mathrm{SiO}_{2}$ layer as a function of temperature over the range $20-300 \mathrm{~K}$ by ps acoustics. We use a pump-probe technique, analog to that in Ref. 28 with a broadband pulse centered at the intermediate frequency of $120 \mathrm{GHz}$. The hypersound pulse generated by a pump laser pulse travels back and forth through the silica layer sandwiched between a thin metallic film and a substrate. Attenuation is evaluated from an analysis of the whole acoustic signal based on a mismatch model including an effective law for the $\omega$ dependence of $\alpha$ in the $\mathrm{SiO}_{2}$ layer. Section II describes the samples used and explains the experimental methods. The variations of sound dispersion and attenuation as a function of $T$ found for the $\mathrm{SiO}_{2}$ layer are presented in Section III. They are discussed in terms of TAR and anharmonicity mechanisms. The number density of TAR defects in the $\mathrm{SiO}_{2}$ layer can be compared to the one of bulk v- $\mathrm{SiO}_{2}$. Section IV concludes the paper.

\section{MATERIAL AND METHODS}

\section{A. Experimental setup}

Sound attenuation in amorphous $\mathrm{SiO}_{2}$ layers is investigated by means of picosecond acoustics. The method is based on the decay analysis of acoustic echoes which go back and forth through a silica layer. The sample is a silica layer grown on a sapphire substrate with an aluminum thin film transducer deposited on top as sketched in the inset of Fig. 2. The set-up is based on a femtosecond Ti:sapphire mode-locked laser providing 80 fs pulses ( $\sim 200$ fs pulses incoming on the sample) and operating at $\sim 770 \mathrm{~nm}$ with a repetition rate of $80 \mathrm{MHz}$. Each pump laser pulse, focused on the $\mathrm{Al}$ film, causes it to rapidly heat. As a result, a strain wave packet is launched into the sample and propagates through the 


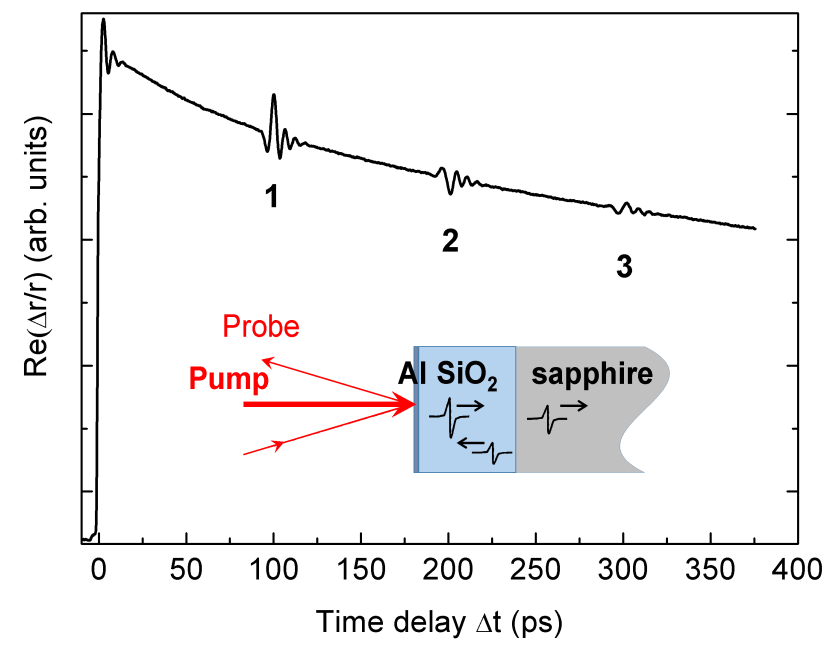

FiguRE 2. (color online) Transient change of reflectivity (real part) at $45 \mathrm{~K}$ as a function of time delay (sputtered- $\mathrm{SiO}_{2}$ sample). The three echoes, labeled according to their number of round trip, appear on top of a slow relaxation. A schematic sketch of the experiment is shown in inset: the pump pulse is absorbed by the Al film; an acoustic pulse is generated, and propagates through the silica layer as suggested by the arrow; the pulse is partly reflected at $\mathrm{SiO}_{2}$ /sapphire interface and returns to the $\mathrm{Al}$ film in which it is detected by the probe pulse.

$\mathrm{SiO}_{2}$ layer. The strain wave is mainly longitudinal as the pump beam is focused over a spot whose diameter is large (about $60 \mu \mathrm{m}$ wide) compared to the thinness of the Al film (about $20 \mathrm{~nm}$ thick). The strain pulse is partly reflected at the $\mathrm{SiO}_{2}$ /sapphire interface. On returning to the $\mathrm{Al}$ layer, the strain pulse induces free surface and $\mathrm{SiO}_{2}$ interface displacements as well as local modification of the $\mathrm{Al}$ optical constants. Both effects lead to a transient change of the reflectivity $\Delta r, r=\rho e^{i \phi}$ being the reflection coefficient of the electric field. The changes of amplitude and phase ( $\Delta \rho$ and $\Delta \phi$ respectively) can be measured thanks to a Sagnac interferometer ${ }^{10}$ which is particularly insensitive to low frequency noise induced by its mechanical components. The pump is modulated at $1 \mathrm{MHz}$ to allow synchronous detection through a lock-in amplifier and reach a sensitivity better than $10^{-6}$.

The sample is mounted in a He continuous-flow cryostat to carry out the measurements from $15 \mathrm{~K}$ to $300 \mathrm{~K}$. Because of the low thermal conductivity of v-SiO 2 , the optical power has been set in order to avoid a steady-state heating of the sample. Practically, we checked that the measured echoes scale linearly with pump and probe power. Typical powers are few tens of $\mathrm{mW}$ for the pump and $1 \mathrm{~mW}$ to $2 \mathrm{~mW}$ for the probe. Both beams are focused to a spot diameter of $60 \mu \mathrm{m}$ on the sample surface.

A typical $\frac{\Delta \rho}{\rho}$ signal measured at $45 \mathrm{~K}$ is shown in Fig. 2. The absorption of the pump pulse in the Al film gives rise to the sudden increase in the signal at $\Delta t=0$ due to the rapid increase of the metallic film temperature. At later time delays, three transient features are recorded on top of a slow decaying background. The latter is the sign of the Al film cooling as heat flows away from the detection area. The subtraction of this background leaves a series of $n$ acoustic echoes, shown in Fig. 3 as black dots, corresponding to the strain pulse detection in the $\mathrm{Al} \mathrm{film}$ after propagating back and forth $n$ times through the $\mathrm{SiO}_{2}$ layer. Each echo is labeled according to its number of round trip $n$. For thin Al films, the spectral content of the strain pulse is mainly set by the film thickness. ${ }^{28,34}$ It extends from about $40 \mathrm{GHz}$ to $200 \mathrm{GHz}$ as shown in Fig 3(b). Studying the changes of shape of the successive echoes allows to evaluate the sound-attenuation coefficient of the $\mathrm{SiO}_{2}$ layer within the frequency band of the pulse.

\section{B. Samples}

The physical characteristics of deposited amorphous $\mathrm{SiO}_{2}$ layers can be, in practice, different from those of bulk fused silica, depending on deposition techniques and growth conditions. ${ }^{35,36}$ Here, the $\mathrm{SiO}_{2}$ layer is prepared by the reactive radio frequency $(\mathrm{RF})$ magnetron sputtering technique. Our first stage has been to optimize the deposition parameters so that properties (as density, refractive index and sound velocity) of the produced $\mathrm{SiO}_{2}$ layer resemble those of thermally grown $\mathrm{SiO}_{2}$ on silicon taken as a reference. The selected deposition conditions are summarized in Tab. I. The sapphire surface has to be cleaned by hydrogen plasma etching before $\mathrm{SiO}_{2}$ deposition. The thickness of the selected $\mathrm{SiO}_{2}$-layer evaluated by ellipsometry is $d=281(2) \mathrm{nm}$. The $\mathrm{OH}$ content in the $\mathrm{SiO}_{2}$-layer is expected to be the one of the target, i.e. $\leq 20 \mathrm{ppm}$. Finally, the layer is capped by an evaporated thin Al film of nominal thickness $l=20 \mathrm{~nm}$. Aluminum is chosen for short optical skin depth enabling broadband detection. Its large linear dilatation coefficient induces also efficient photo-thermal transduction. Moreover its acoustic impedance is close to the silica one thus reducing reflection at $\mathrm{Al} / \mathrm{SiO}_{2}$ interface. Sapphire, in contrast, is chosen on account of its high acoustic impedance as compared to silica one. This results in the large strain pulse reflection, $r_{\mathrm{ac}} \simeq 0.53$, at $\mathrm{SiO}_{2} /$ sapphire interface.

TABLE I. $\mathrm{SiO}_{2}$ film deposition parameters

\begin{tabular}{ll}
\hline Target & fused quartz \\
Gas composition & $50 \% \mathrm{Ar}+50 \% \mathrm{O}_{2}$ \\
Sputtering pressure & $0.2 \mathrm{~Pa}$ \\
RF power & $1.1 \mathrm{~W} / \mathrm{cm}^{2}$ \\
Substrate temperature & $300^{\circ} \mathrm{C}$ \\
Deposition rate & $0.115 \mu \mathrm{m} / \mathrm{h}$ \\
\hline
\end{tabular}




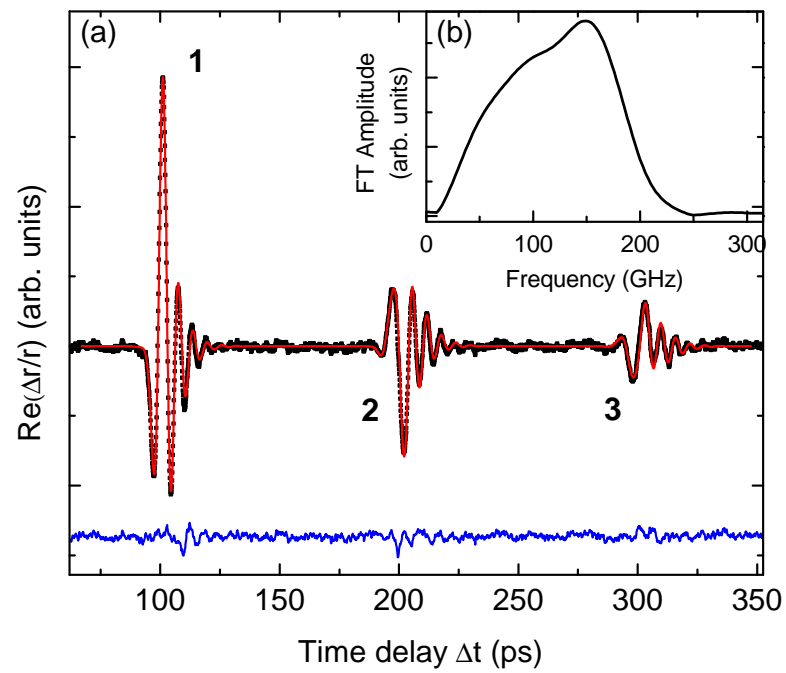

Figure 3. (color online) (a) Transient change of reflectivity (real part) at $45 \mathrm{~K}$ as a function of time delay, after subtraction of an ad-hoc smooth thermal background $(\bullet)$ to enhance the echoes (sputtered-SiO $\mathrm{S}_{2}$ sample). Superimposed, typical fit (red line) of the three first acoustic echoes. Bottom curve: difference between the experimental signal and the fit (residual), shifted for clarity. (b) Fourier transform amplitude of the first echo.

To provide a benchmark sample, we also produce a thermally grown $\mathrm{SiO}_{2}$ layer by dry thermal oxidation of silicon. The oxidation is performed at a substrate temperature of $1100{ }^{\circ} \mathrm{C}$ in a flow of $\mathrm{O}_{2}$ gas during about 8 hours. This results in a $\mathrm{SiO}_{2}$-layer about $400(10) \mathrm{nm}$ thick. An Al-film $20 \mathrm{~nm}$ thick is evaporated on top as above. This sample is referred to as dryox hereafter.

\section{Data analysis}

We are interested in computing the acoustic echo signal which is associated to the propagating part of the photo-thermal strain generated in the Al film. The signal is analyzed in the time domain with the help of an acoustic impedance mismatch model as discussed in the forthcoming paragraph. As introduced in Section I, TAR and anharmonicity mechanisms are expected to damp the acoustic pulse in the $\mathrm{SiO}_{2}$-layer. Both mechanisms can be described in terms of relaxation processes. However these have different $\omega$ and $T$ dependences. We thus employ an ad hoc power law for the frequency dependence of sound attenuation in the layer, $\alpha(\omega, T)=\alpha_{0}(T) \omega^{p(T)}$ in order to represent the effective sound damping over the $\omega$-band of the pulse. ${ }^{7}$ The exponent should be $T$-dependent while satisfying $p(T) \leq 2$. In the following, $\alpha$ denotes the rate of attenuation of the energy in the acoustic pulse, per unit distance.

Strain-pulse generation from thermal expansion of metallic films upon light-pulse absorption is welldocumented. ${ }^{37,38}$ The photo-thermal generation is treated as an impulsive surface loading with a Gaussian profile $\sigma(t, z=0) \propto e^{-t^{2} / \delta^{2}}$, whose typical duration is characterized by $\delta, z$ being the coordinate perpendicular to the sample. Free boundary conditions at the Al freesurface and displacement-stress continuity at the other two interfaces are assumed. Let us also consider the sapphire substrate as an infinite medium. Based on an acoustic impedance mismatch model, one can calculate the $\mathrm{Al}$ free-surface displacement, $u_{0}(\omega)$, from the transient surface stress $\sigma(t, z=0)$ :

$$
u_{0}(\omega)=\frac{A(\omega) e^{-i q_{m} l}+B(\omega) e^{i q_{m} l}}{A(\omega) e^{-i q_{m} l}-B(\omega) e^{i q_{m} l}} \frac{\sigma(\omega, z=0)}{i Z_{m} v_{m} q_{m}}
$$

with

$$
\begin{aligned}
A(\omega)= & {\left[1+\frac{Z_{g}}{Z_{m}}\left(1+\frac{\alpha}{2 i q_{g}}\right)+\frac{Z_{s}}{Z_{g}}\left(1+\frac{\alpha}{2 i q_{g}}\right)^{-1}+\frac{Z_{s}}{Z_{m}}\right] e^{-i q_{g} d} e^{\frac{-\alpha d}{2}} } \\
+ & {\left[1-\frac{Z_{g}}{Z_{m}}\left(1+\frac{\alpha}{2 i q_{g}}\right)-\frac{Z_{s}}{Z_{g}}\left(1+\frac{\alpha}{2 i q_{g}}\right)^{-1}+\frac{Z_{s}}{Z_{m}}\right] e^{i q_{g} d} e^{\frac{\alpha d}{2}} } \\
B(\omega)= & {\left[1-\frac{Z_{g}}{Z_{m}}\left(1+\frac{\alpha}{2 i q_{g}}\right)+\frac{Z_{s}}{Z_{g}}\left(1+\frac{\alpha}{2 i q_{g}}\right)^{-1}-\frac{Z_{s}}{Z_{m}}\right] e^{-i q_{g} d} e^{\frac{-\alpha d}{2}} } \\
+ & {\left[1+\frac{Z_{g}}{Z_{m}}\left(1+\frac{\alpha}{2 i q_{g}}\right)-\frac{Z_{s}}{Z_{g}}\left(1+\frac{\alpha}{2 i q_{g}}\right)^{-1}-\frac{Z_{s}}{Z_{m}}\right] e^{i q_{g} d} e^{\frac{\alpha d}{2}} }
\end{aligned}
$$

where $v, q$ and $Z$ are sound velocity, wave number and impedance in the medium specified in subscript $(m$ stands for Al-metal, $g$ for $\mathrm{SiO}_{2}$-glass and $s$ for sapphire- substrate); $l$ and $d$ denote the thicknesses of the metallic Al-film and the glassy $\mathrm{SiO}_{2}$-layer, respectively.

Two distinct processes act on the reflection coefficient 
of the multilayer system: first, the elastic strain slightly modifies the optical properties within the layers and, second, the interfaces of the sample are displaced by the acoustic field. ${ }^{39,40}$ However, as most of the probe light is either reflected by or absorbed in the capping metallic layer, the photo-elastic response from both the $\mathrm{SiO}_{2}$ layer and the substrate can be neglected. Finally (see details in the Supplemental Material ${ }^{41}$ ), the real part of the reflectivity changes are dominated by the Al-film thickness changes alone, and may be given by:

$$
\begin{aligned}
\frac{\Delta \rho(\omega)}{\rho} & =\operatorname{Re}\left\{2 i k_{0}\left[\varepsilon_{m}+\frac{1}{2} \frac{\partial \varepsilon_{m}}{\partial \eta}\right]\right. \\
& \left.\times \frac{a_{m} b_{m}}{n_{0} a_{0} b_{0}}\left[\cos \left(q_{m} l\right)-1\right] u_{0}(\omega)\right\}
\end{aligned}
$$

where the subscript 0 stands for air; $k$ is the electromagnetic field wave number; $a$ and $b$ are the amplitudes of incident and reflected electromagnetic fields, respectively; $\varepsilon$ is the dielectric constant and $\frac{\partial \varepsilon}{\partial \eta}$ is its variation with strain; $q$ is the acoustic wave number. A Fourier transform of Eq. 5 gives the calculated change of reflectivity in the time domain, and $\frac{\Delta \rho(t)}{\rho}$ is adjusted to the measured echoes to assess acoustic attenuation within the $\mathrm{SiO}_{2}$-layer.

The details of the fitting procedure are presented in the Supplemental Material. ${ }^{41}$ In the first place, the $T$ independent parameters entering the mismatch model are assessed by analyzing the shape of the first echo at $15 \mathrm{~K}$ for which the reflection losses dominate alone. In this respect, the surface-loading duration is found to be $\delta=1.6 \mathrm{ps}$ and the metallic Al-film thickness $l=18.8 \mathrm{~nm}$ is evaluated. The acoustic impedance of the $\mathrm{SiO}_{2}$ layer and the one of the substrate are set to their nominal value, whereas the Al-film acoustic impedance needs to be slightly adjusted. The best value is found to be 1.1 times the Al bulk value, possibly due to a partial oxydation of the film. Then, the time delay $\Delta t=d / v_{g}$ between successive echoes for each of the data (recorded at different temperatures) can be adjusted. Assuming $v_{g}=5.95 \mathrm{~nm} / \mathrm{ps}$ the resulting values of $d$ range from $283.2 \mathrm{~nm}$ to $285.0 \mathrm{~nm}$ in line with ellipsometry characterization. Since the thermal expansion is negligible, the $\Delta t(T)$ variations actually reflect sound velocity variations such that $\frac{\Delta v_{g}}{v_{g}} \lesssim 1 \%$ over the whole investigated $T$ range. Finally, all the above parameters being fixed, the attenuation-law parameters $\left(\alpha_{0}\right.$ and $p$ ) are obtained by fitting the three successive echoes to the model using a least squares method. Assuming only white noise over the signals, one obtains the standard deviation on parameters from which the 99 percent confidence interval may be derived. A typical signal at $45 \mathrm{~K}$ and its best fit are displayed in Fig. 3, exhibiting a very good agreement as shown by the small residual (bottom curve). Additional typical fitted curves for some selected $T$ from $15 \mathrm{~K}$ to $300 \mathrm{~K}$ are shown in the Supplemental Material. ${ }^{41}$ The ability to carry out a simultaneous analysis of the first three echoes appears to be decisive in evaluating accurate attenuation parameters.
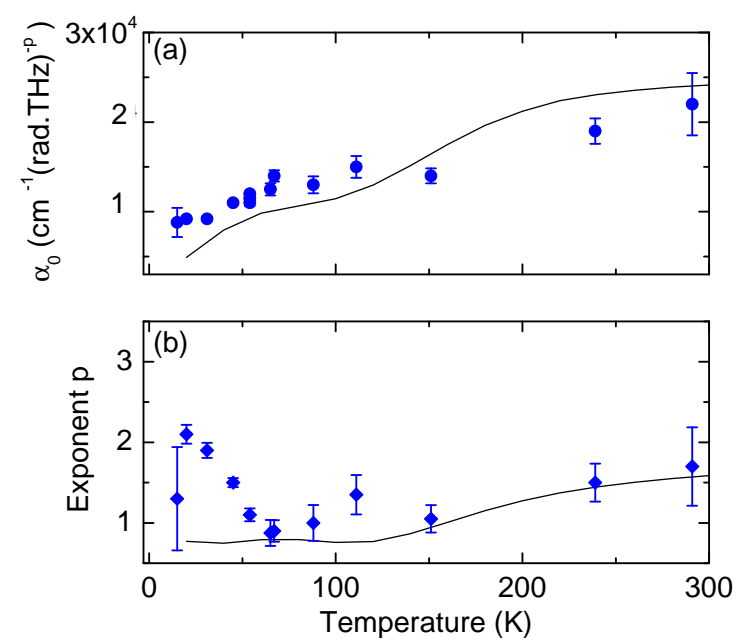

Figure 4. (color online) Parameters $\alpha_{0}$ and $p$ entering the local effective law, $\alpha(\omega, T)=\alpha_{0}(T) \omega^{p(T)}$, for acoustic attenuation over the $\omega$-band of the pulse. The lines are expectation from a model combining damping from anharmonicity and $1.5 \times$ TAR defects as explained in text.

Uncertainty on the attenuation values are calculated by propagating the confidence limits on the fitted parameters to the $\alpha$-values. The true uncertainty might be, however, somewhat larger due to the shortcomings of the model used for analyzing the echoes (Gaussian thermal strain generation in the $\mathrm{Al}$ film, monolayer of $\mathrm{Al}$ metal, perfect bounding at interfaces, etc.) which can hardly be quantified.

The above procedure cannot be applied to the dryox sample due to the weak acoustic contrast between v- $\mathrm{SiO}_{2}$ and silicon. In that case, the second echo is poorly detected. Sound velocities $v(T)$ for the dryox $\mathrm{SiO}_{2}$-layer are nevertheless determined evaluating the round-trip time for the first echo.

\section{RESULTS AND DISCUSSIONS}

\section{A. Acoustic attenuation}

The coefficients $\alpha_{0}$ and $p$ found upon cooling from $300 \mathrm{~K}$ to $15 \mathrm{~K}$ are shown in Figs. 4(a) and 4(b). The prefactor $\alpha_{0}$ decreases by a factor of about 2.5 emphasizing the lowering of the acoustic attenuation with decreasing $T$. The exponent $p$ is found $\leq 2$ at all temperatures which is consistent with relaxation processes. The resulting attenuation rates agree with the available ps acoustics results at room $T$ for $\mathrm{SiO}_{2}$ layers as illustrated in Fig. 5(a). We also plot in Fig. 5(b) the temperature dependence of the attenuation rate found at $33.5 \mathrm{GHz}(\mathbf{\Lambda})$ for which there exists a precise evaluation of $\alpha(T)$ in bulk v- $\mathrm{SiO}_{2}$ from Brillouin scattering measurements ${ }^{12,42}$ to serve as a reference. The data roughly overlap each other. Before we go any further, we outline in the following paragraph 

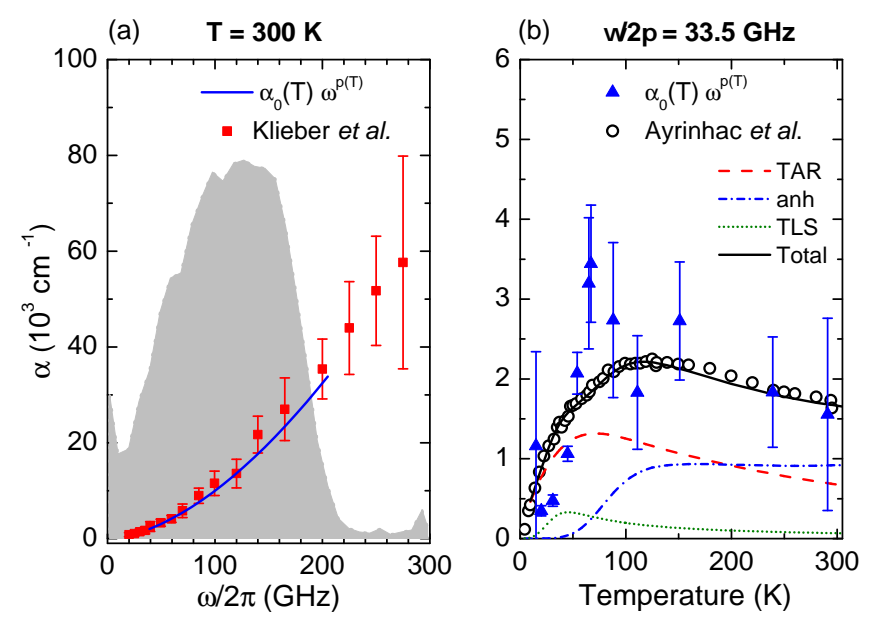

Figure 5. (color online) (a) The acoustic attenuation rate as a function of frequency $(-)$ derived from the $\alpha_{0}$ and $p$ parameters found at $300 \mathrm{~K}$. The shaded area reminds the power spectrum of the acoustic pulse (first echo). Our finding overlaps the experimental determination by Klieber et al. ${ }^{27}$ at room temperature ( $\mathbf{\square})$. (b) The attenuation rates as a function of temperature derived at $33.5 \mathrm{GHz}$ from the obtained $\alpha_{0}(T)$ and $p(T)$ parameters $(\mathbf{\Lambda})$. The values found in bulk $\mathrm{v}-\mathrm{SiO}_{2}$ from Brillouin scattering measurements at the same frequency (o) are shown as a reference ${ }^{42}$. The solid line is an adjustment of the Brillouin data made in Refs. 12 and 42, which is the sum of two main contributions, $\alpha_{\mathrm{TAR}}$ and $\alpha_{\text {anh }}$ plus the tiny contribution $\alpha_{\mathrm{TLS}}$ as explained in text.

how the $T$ dependence of the attenuation coefficient is currently explained. Note that attenuation coefficient is proportional to internal friction, i.e. the inverse of quality factor $Q^{-1}=\alpha v / \omega$, a form that will be used hereafter.

As already pointed out in the introduction, the hypersonic attenuation in $\mathrm{v}-\mathrm{SiO}_{2}$ mainly originates from the combined effects of TAR and anharmonicity. The phenomenological TAR model assumes the occurrence of structural defects that are represented by particles relaxing in an asymmetric double-well potential. It is widely recognized that sonic and ultrasonic damping in $\mathrm{v}-\mathrm{SiO}_{2}$ at $T$ above $10 \mathrm{~K}$ is fully dominated by TAR defects. $6,43,44$ The acoustic waves couple to the defects by a deformation potential $\gamma$, modifying their equilibrium population. Broad distributions for both energy barriers $V$ and potential asymmetry $\Delta$ must be taken into account. The thermal rate of jumping from one state to the other (i.e. the inverse of relaxation time) for a given defect follows from Arrhenius expression,

$$
\tau^{-1}=\frac{\tau_{0}^{-1}}{2} \exp \left(-\frac{V}{k_{\mathrm{B}} T}\right) \cosh \left(\frac{\Delta}{2 k_{\mathrm{B}} T}\right)
$$

where $\tau_{0}^{-1}$ is an attempt frequency. Integrating over the distribution one obtains the expression,

$$
\begin{aligned}
& Q_{\mathrm{TAR}}^{-1}(\omega, T)=\frac{\gamma^{2}}{\rho v^{2} T} \int_{-\infty}^{\infty} \mathrm{d} \Delta \int_{0}^{\infty} \mathrm{d} V P(\Delta, V) \\
& \times \operatorname{sech}^{2}\left(\frac{\Delta}{2 k_{\mathrm{B}} T}\right) \frac{\omega \tau}{1+\omega^{2} \tau^{2}}
\end{aligned}
$$

where $P(\Delta, V) \mathrm{d} \Delta \mathrm{d} V$ is the density of double well entities. A product of two distribution functions, a Gaussian in $\Delta, f(\Delta) \propto \exp \left(-\Delta^{2} / 2 \Delta_{\mathrm{C}}^{2}\right)$ of width $\Delta_{\mathrm{C}}$, times a modified Gaussian $g(V) \propto\left(V / V_{0}\right)^{-\zeta} \exp \left(-V^{2} / 2 V_{0}^{2}\right)$ of width $V_{0}$ is generally assumed ( $\zeta$ is fixed to $\left.1 / 4\right) .{ }^{45}$ Introducing the latter in Eq. 7 leads to a functional form for $Q_{\mathrm{TAR}}^{-1}$ in which the total density of relaxing defects,

$$
N \equiv \int_{-\infty}^{\infty} \mathrm{d} \Delta \int_{0}^{\infty} \mathrm{d} V P(\Delta, V)
$$

enters as a prefactor. A dimensionless constant $\mathcal{C}_{\mathrm{TAR}} \equiv$ $\frac{N_{\mathrm{g}} \gamma^{2} N}{V_{0} \rho v^{2}}$ is actually introduced for convenience (the constant of integration $N_{\mathrm{g}}$ equals 1.54 for $\zeta \simeq 0.25$ ). The handling of these equations is described in great details in Ref. 12. The set of parameters $V_{0} / \Delta_{\mathrm{C}}=8.2$ with $V_{0} / k_{\mathrm{B}}=659 \mathrm{~K}, \tau_{0}=10^{-12.2} \mathrm{~s}$ and $\mathcal{C}_{\mathrm{TAR}}=1.9 \times 10^{-3}$ was determined for $\mathrm{v}-\mathrm{SiO}_{2}$ by fitting internal friction and velocity dispersion results over sonic and ultrasonic domains. The same values apply to both LA and TA modes over four decades of frequency. The TAR contribution to $\alpha(T)$ extrapolated at the hypersonic frequency $33.5 \mathrm{GHz}$ from these parameters is plotted as a dashed line in Fig. 5(b). A minor contribution to $\alpha$ from the tunneling systems (TLS) via relaxation channels so-called "incoherent tunneling" is also added from Ref. 12. This contribution is shown in Fig. 5(b) as a thin doted black line.

The residual attenuation is interpreted as resulting from network viscosity. ${ }^{12,46}$ The same approach as the one developed for crystals by Maris ${ }^{11}$ is used for glasses. Phonons are treated as a gas in which anharmonic interactions lead to a mean thermal lifetime $\tau_{\text {th }}(T)$ between collisions. ${ }^{47}$ This leads to the internal friction,

$$
Q_{\mathrm{anh}}^{-1}(\omega, T)=A \frac{\omega \tau_{\mathrm{th}}}{\left(1+\omega^{2} \tau_{\mathrm{th}}^{2}\right)}
$$

with

$$
A(T)=\frac{C_{\mathrm{v}} T v}{2 \rho v_{\mathrm{D}}^{3}}{\overline{\gamma_{\mathrm{G}}}}^{2} .
$$

Here, $C_{\mathrm{v}}$ is the specific heat per unit volume, $v_{\mathrm{D}}$ is the Debye velocity, and $\overline{\gamma_{\mathrm{G}}}$ is an average Grüneisen parameter. The strongest assumptions in this development are the use of a single relaxation time $\tau_{\text {th }}(T)$ for thermal modes and a constant $\overline{\gamma_{\mathrm{G}}}$. The anharmonic contribution to $\alpha(T)$ evaluated in Ref. 12 is plotted as a dot-dash line in Fig. 5(b) using $\overline{\gamma_{\mathrm{G}}}=3.8$ and the values of $\tau_{\text {th }}$ shown in Fig. 6(b) of Ref. 12.

As can be seen in Fig. 5(b), the attenuation found in the $\mathrm{SiO}_{2}$-layer at $33.5 \mathrm{GHz}$ displays a $T$-dependent behavior which follows the same trend as that of bulk v-SiO 2 

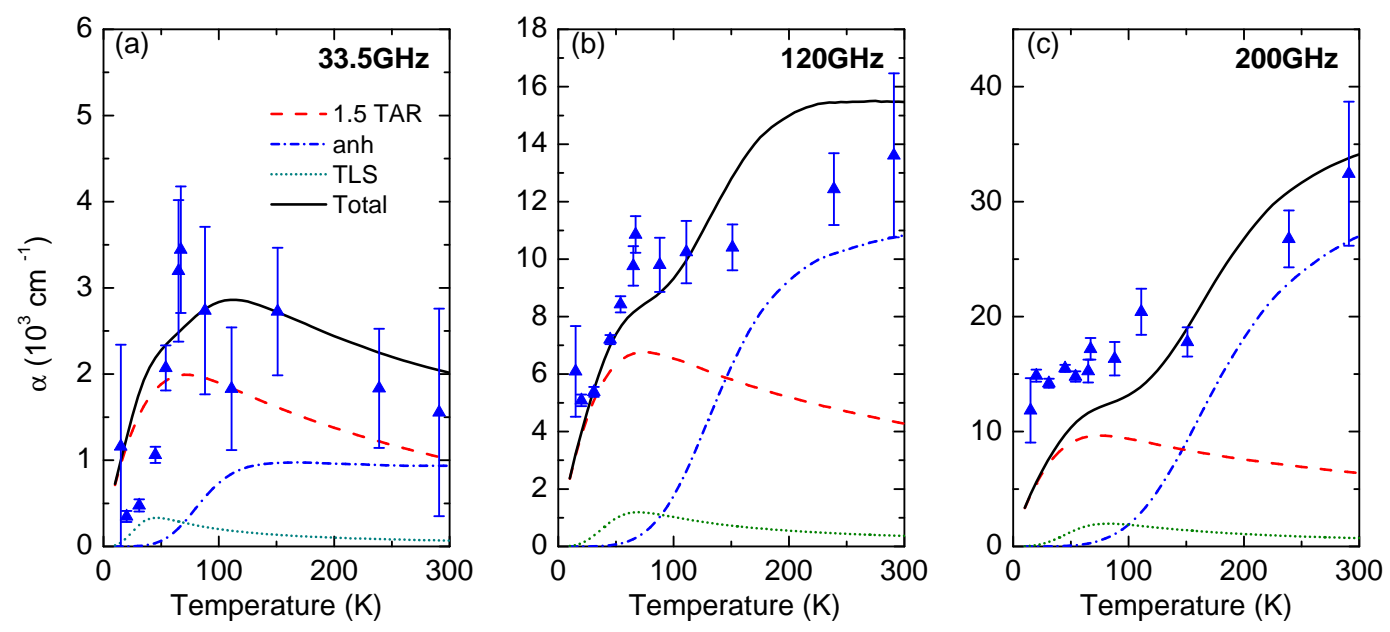

Figure 6. (color online) Acoustic attenuation coefficient of energy obtained for the $\mathrm{SiO}_{2}$ layer at $33.5 \mathrm{GHz}$ (a), $120 \mathrm{GHz}$ (b) and $200 \mathrm{GHz}$ (c) in this work. The values are derived from the fitted parameters shown in Fig. 4 as explained in text. The solid curves are predictions combining two main contributions, $1.5 \times \alpha_{\mathrm{TAR}}$ and $\alpha_{\mathrm{anh}}$ plus the tiny contribution $\alpha_{\mathrm{TLS}}$. The $\alpha_{\mathrm{TAR}}$ contribution in the $\mathrm{SiO}_{2}$ layer is found nearly 1.5 times that in $\mathrm{v}-\mathrm{SiO}_{2}$ indicating more numerous relaxing defects.

at the same frequency. However, a detailed inspection yields to the following two observations:

(i) The attenuation in the $\mathrm{SiO}_{2}$ layer exhibits a peak at about $50 \mathrm{~K}$, just as that of bulk v- $\mathrm{SiO}_{2}$ but it appears slightly larger and sharper. According to the TAR model, this would indicate the occurrence of more numerous relaxing defects in the layer than in the bulk sample.

(ii) Near room $T$, in contrast, the attenuation of the $\mathrm{SiO}_{2}$ layer is mainly similar to that of bulk v- $\mathrm{SiO}_{2}$ within the experimental errors. We therefore conclude that damping due to anharmonicity likely remains mostly identical for both materials. It also seems likely that phonon anharmonicity should not depend on the amount of relaxing defects, since they hardly affect the vibrational density of states.

Consequently, we plot as a dashed line in Fig. 6(a) the computed attenuation from TAR by arbitrarily multiplying the total density of relaxing defects by 1.5 (i.e. $1.5 \times \mathcal{C}_{\mathrm{TAR}}=2.85 \times 10^{-3}$ ) while keeping the double-wells parameters, $V_{0}$ and $\Delta_{\mathrm{C}}$, and the frequency attempt, $\tau_{0}$, unchanged. By adding to the latter the contribution from anharmonicity found in $\mathrm{v}-\mathrm{SiO}_{2}$, one obtains the curve shown in Fig. 6(a) as a solid line. This almost fits to the data in the $\mathrm{SiO}_{2}$ layer.

The same model (one and a half TAR-defects plus anharmonicity) approximately matches both the high frequency data at $120 \mathrm{GHz}$ and $200 \mathrm{GHz}$ also shown in Fig. 6 without any additional assumptions. The two mechanisms are observed to clearly separate with increasing $\omega$, the anharmonic part growing faster than the TAR one. Indeed, from Eq. 1, TAR attenuation near the maximum of its peak grows linearly with increasing $\omega, \alpha_{\mathrm{TAR}} \propto \omega$. In contrast, anharmonicity is in the regime $\omega \tau_{\text {th }} \ll 1$, so that $\alpha_{\text {anh }} \propto \omega^{2}$. All the above explains a posteriori the non-trivial $T$-dependent behavior found for the exponent of the power-law, used to represent the $\omega$-dependence of attenuation within the pulse and shown in Fig 4(b). The calculated $p$ from the model ( $1.5 \times$ TAR plus anh.) is also shown in Fig 4(b) as a solid line. Anharmonicity damping dominates the $p(T)$ variations near room $T$ while TAR mechanisms should prevail at low $T$. The deviation below $50 \mathrm{~K}$ might possibly be due to a small contribution of the additional strong attenuation mechanism $\left(\alpha \propto \omega^{4}\right)$ expected to come into play at high frequencies over anharmonicity as mentioned in the Introduction. Lastly, we should remark that the overall analysis further demonstrates the operability of the treatment proposed for network viscosity in Ref. 12 . Here we show that this remains suitable up to at least $200 \mathrm{GHz}$.

The number density of relaxing defects may be evaluated from the amplitude of TAR losses $\left(i . e\right.$. from the $\mathcal{C}_{\text {TAR }}$ value). For $\mathrm{v}_{-} \mathrm{SiO}_{2}$, we get $0.64 \times 10^{-12} \mathrm{~J} \mathrm{~m}^{-3}$ for $\gamma^{2} N$, the defects density multiplied by the square of the deformation potential. Taking $\gamma$ of the order of $1 \mathrm{eV}$ leads to about 1 defect per $650 \mathrm{SiO}_{2}$ molecules, close to the value $1 / 500$ given in Ref. 48. Microscopic models have been proposed to explain the origin of these relaxing entities. ${ }^{5}$ The role of impurity defects (including that of $\mathrm{OH}$ group) was early excluded since they hardly affect acoustic loss amplitude. ${ }^{49}$ The basic assumption is that owing to disorder, atoms or groups of atoms can occupy two or more equilibrium configurations. Applied pressure strongly affects TAR parameters. ${ }^{42,44}$ It is further observed that TAR processes completely vanish in permanently densified silica glass upon $20 \%$ compaction. ${ }^{46,50,51}$ This suggests that the relaxing entities are closely related to the largest interstitial voids within $\mathrm{SiO}_{2}$ network (highmembered rings) which are removed by compaction. ${ }^{42}$ The latter are manifested in the medium-range structure via the broad distribution of inter-tetrahedral bonding. ${ }^{52}$ Compaction sharpens this distribution ${ }^{53}$ thereby suppressing many double wells. Recent numerical works pro- 


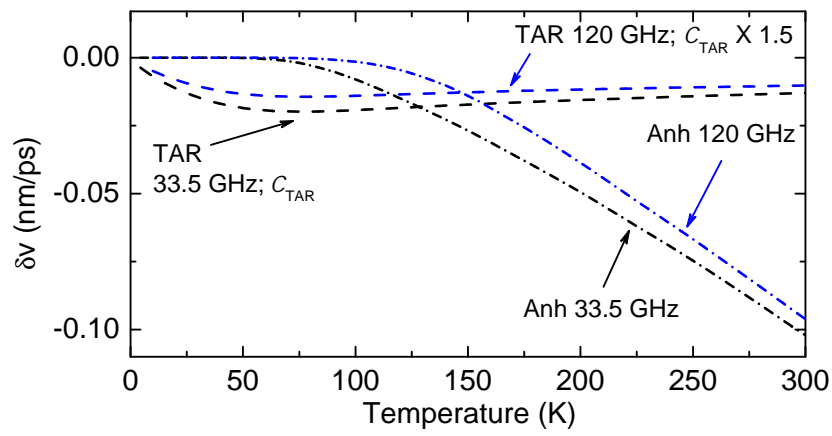

Figure 7. (color online) The expectation for the $\delta v(T)$ patterns associated with TAR (dashed lines) and anharmonicity (dash-dot lines) mechanisms found in damping. The computation is at $120 \mathrm{GHz}$ for the $\mathrm{SiO}_{2}$-layer (blue lines) while it is at $33.5 \mathrm{GHz}$ for bulk v-SiO 2 (black lines).

vide new insights into the nature of the relaxing entities in $\mathrm{SiO}_{2}$ glass. ${ }^{54,55}$ They would mainly involve rotations of the oxygen bonds over several connected tetrahedrons. These motions might indeed be promoted adjacent to the network vacancies. The enhancement of TAR absorption in deposited $\mathrm{SiO}_{2}$ layer should thus indicate enhanced topological disorder that would promote the formation of numerous double-wells (we cannot however completely exclude either an enhanced value of $\gamma$ possibly due to internal constraints in the $\mathrm{SiO}_{2}$ layer). Anyway, this must be obviously related to layer preparation.

\section{B. Velocity variations}

The expectation for the velocity changes $(\delta v / v)_{\mathrm{TAR}}$ and $(\delta v / v)_{\text {anh }}$ associated with the damping processes found in attenuation can be easily calculated. ${ }^{12}$ Velocity dispersion being very low over the frequency spread of the strain pulse, we consider here the mean frequency of $120 \mathrm{GHz}$ ( $v$ is incidentally assumed to be frequency independent in the fitting procedure). The reference value $v_{0}$ in the differential quantity $\frac{\delta v}{v}=\frac{v-v_{0}}{v_{0}}$ is taken as the low- $T$ limit of $v$ found by Brillouin scattering for $\mathrm{v}_{-} \mathrm{SiO}_{2}$, namely $v_{0}=5.882 \mathrm{~nm} \mathrm{ps}^{-1}$. Applying the Kramers-Kronig relation to Eq. 9 gives $^{12}$ :

$$
(\delta v / v)_{\mathrm{anh}}=-\frac{A}{2} \frac{1}{1+\omega^{2} \tau_{\mathrm{th}}^{2}}
$$

This produces the $T$-dependence of $\delta v_{\text {anh }}$ shown in Fig. 7 as dash-dot lines for $33.5 \mathrm{GHz}$ and $120 \mathrm{GHz}$. The $(\delta v / v)_{\text {anh }}$ variations with $\mathrm{T}$ are controlled by $\tau_{\mathrm{th}}(T)$ variations. The knee in the curves around $150 \mathrm{~K}$ marks the region where $\omega \tau_{\text {th }} \simeq 1$. It occurs at slightly larger $T$ for $120 \mathrm{GHz}$ than for $33.5 \mathrm{GHz}$. Below the knee, $\delta v_{\text {anh }}(T)$ tends to zero since $\omega \tau_{\text {th }} \gg 1$. Beyond it, $\delta v_{\text {anh }}$ becomes mostly controlled by the increase of $A(T)$ leading to an $\omega$-independent linear decrease with $T$ as $\omega \tau_{\text {th }} \ll 1$.

The expected TAR contribution to velocity changes, $\delta v_{\mathrm{TAR}}$, is calculated using Eq. 1(b) of Ref. 12 with the pa-

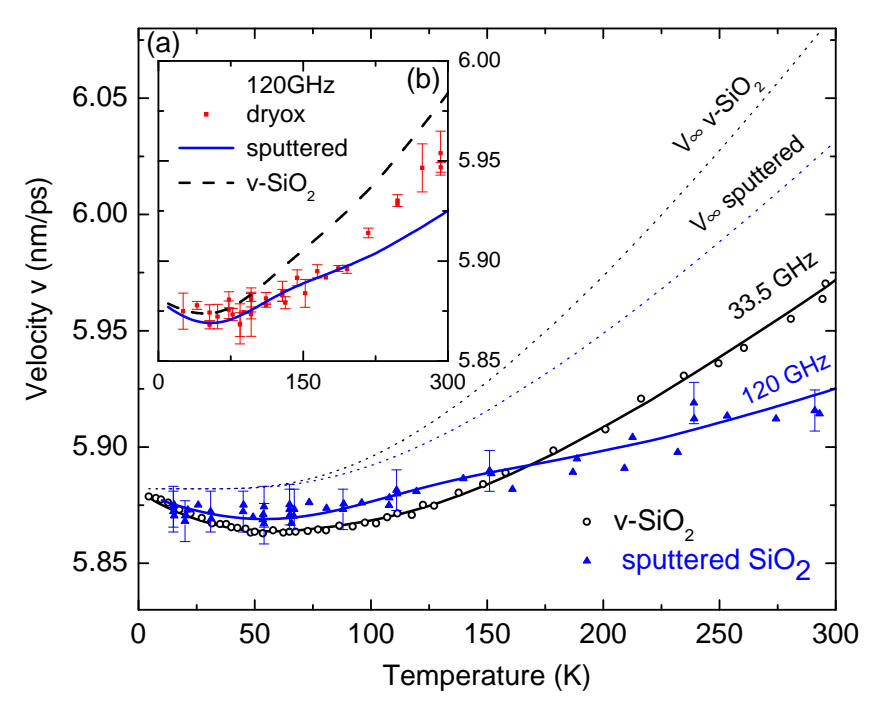

Figure 8. (color online) (a) The $v(T)$ for the $\mathrm{SiO}_{2}$ layer are shown as triangles ( $)$. The data points with error bars are from the fitting procedure, the ones without are extra data from the round-trip time method. The $v(T)$ values for bulk v$\mathrm{SiO}_{2}$ at $33.5 \mathrm{GHz}$ shown as open dots (०) serve as a reference. The solid lines are our calculation $v=v_{\infty}+\left(\delta v_{\mathrm{TAR}}+\delta v_{\text {anh }}\right)$ using the appropriate $\delta v_{\mathrm{TAR}^{-}}$and $\delta v_{\text {anh-values from Fig. } 7 \text { and }}$ the special purpose unrelaxed velocities $v_{\infty}$ shown as dotted lines. (b) The inset shows the $v(T)$ values found for the $d$ ryox $\mathrm{SiO}_{2}$-layer from the round trip time method $(\bullet)$. They fall in between the $v(T)$ curves for the sputtered layer (lower blue curve) and for bulk v-SiO ${ }_{2}$ computed at $120 \mathrm{GHz}$ (upper black line).

rameters describing attenuation data found in the $\mathrm{SiO}_{2}$ layer. Owing to the Kramers-Kronig relation, $(\delta v / v)_{\mathrm{TAR}}$ is expressed by an integral similar to Eq. 7, in which the last term is replaced by $-\frac{1}{2} \frac{1}{1+\omega^{2} \tau^{2}}$. This produces the $T$ dependence of $\delta v_{\text {TAR }}$ illustrated in Fig. 7. $\delta v_{\text {TAR }}(T)$ should exhibit a dip nearly in coincidence with the maximum in internal friction $Q_{\mathrm{TAR}}^{-1}(T)$, as already discussed. ${ }^{12,44}$ The absorption peak relates to those defects for which $\tau=\omega^{-1}, \tau$ following an Arrhenius law with temperature. Therefore, an increase in $\omega$ should result in an upper shift of the dip in $\delta v_{\text {TAR }}(T)$. This should also involves a reduction of the dip amplitude as shown in Fig. 8(a) of Ref. 12 (due to the Kramers-Kronig transform, $\delta v_{\text {TAR }}$ integrates over all defects with $\left.\tau \leq \omega^{-1}\right)$. The dip amplitude should remain anyway proportional to the total defects density $N$.

The $v(T)$ variations in the $\mathrm{SiO}_{2}$ layer are obtained by two different methods: one is simply the usual roundtrip time method, the other one is the fitting operation explained in section II C. The values found for the sputtered $\mathrm{SiO}_{2}$ layer are shown in Fig. 8(a) as triangle symbols $(\boldsymbol{\Lambda})$. The data points with error bars are from the fitting method whereas the ones without are extra data from the round-trip time method. Accuracy on the absolute scale of $v(T)$ is limited by uncertainty on the layer thickness. Assuming $2 \mathrm{~nm}$ for the latter leads to about 
$0.04 \mathrm{~nm} / \mathrm{ps}$ uncertainty on $v$. In contrast, accuracy on velocity changes $\delta v(T)$ is much higher since it is only governed by uncertainty on pump-probe delay (about $\Delta t \leq 0.1 \mathrm{ps}$ ). This requires however that no fluctuation in layer thickness happens all along the experiment. The layer being possibly slightly wedge-shaped, a minor rescaling was necessary at each change in laser focusing spot location onto the sample. The $v(T)$ from Brillouin scattering in bulk v-SiO 2 are also displayed in Fig. 8 as circle (o) to serve as a reference. ${ }^{42}$ We arbitrarily chose to make so that the velocity of the $\mathrm{SiO}_{2}$-layer matches the one of bulk v-SiO 2 , at low temperature.

The calculated total changes $\delta v_{\mathrm{TAR}}+\delta v_{\text {anh }}$ discussed above should result in the occurrence of a small dip (at low $T$ ) followed by a linear decrease in the $v(T)$ data. In contrast, the $v(T)$ data exhibit a positive temperature coefficient as shown in Fig. 8(a) for both the $\mathrm{SiO}_{2}$-layer (₫) and bulk v- $\mathrm{SiO}_{2}(\circ)$. This is because silica experiences well-known structural changes with $T$ resulting in the occurrence of static $v(T)$-changes which add to the dynamical ones. However, the calculated dynamical changes $\left(\delta v_{\mathrm{TAR}}+\delta v_{\mathrm{anh}}\right)$ explain the low- $T$ behavior of $v(T)$ data as shown in Fig. 8. The tiny dip in $v(T)$ near $60 \mathrm{~K}$ due to relaxing defects is actually partly buried into the experimental uncertainties. Nevertheless, a locally negative curvature of $v(T)$ is observed in the range $100 \mathrm{~K}$ to $150 \mathrm{~K}$ resulting from the anharmonic losses. The latter result gives the earliest direct evidence of dissipative effects of relaxation via anharmonic interactions on sound velocity. The separate effects of thermally activated relaxations and anharmonicity are clearly demonstrated here in an experiment.

Subtracting both dynamical contributions from the $v(T)$ data, one obtains the so-called unrelaxed velocity $v_{\infty}=v-\left(\delta v_{\mathrm{TAR}}+\delta v_{\mathrm{anh}}\right)$. This bare velocity which should be $\omega$ - and $T$-independent, is found to be a growing function of $T$ in $\mathrm{v}^{-\mathrm{SiO}_{2}}$ as discussed in Refs. 12 and 42. The anomalous positive slope in $v(T)$ is in fact a peculiarity of all glasses with tetrahedral network as early reported. ${ }^{56-58}$ This behavior has been attributed to T-dependent and reversible structural effects. ${ }^{59} \mathrm{~A}$ progressive local polyamorphic transition associated with a redistribution of $\mathrm{Si}-\mathrm{O}-\mathrm{Si}$ bonds angles in configurations where they are more resilient, has been found in simulations. ${ }^{60,61}$ The dotted lines in Fig. 8(a) are fits of $v_{\infty}(T)$ to an ad hoc Wachtman's equation for the $\mathrm{SiO}_{2}$ layer and for ${\mathrm{v}-\mathrm{SiO}_{2}}_{2}$ (from Ref. 12). The positive slope in $v_{\infty}(T)$ appears slightly reduced in the $\mathrm{SiO}_{2}$-layer . It may be an additional indication that further small structural differences remain between the sputtered $\mathrm{SiO}_{2}$-film and bulk vitreous silica.

We plot the $v(T)$ results for the dryox $\mathrm{SiO}_{2}$-layer in Fig 8(b). The values fall in between the ones for the sputtered $\mathrm{SiO}_{2}$-layer (blue solid line) and the $v(T)$ curve computed at $120 \mathrm{GHz}$ for bulk v-SiO ${ }_{2}$ (black dashed line). A small dip at low- $T$ (from TAR defects) followed by a slightly negative curvature (from anharmonic losses) is observed as well. Then the positive slope of $v_{\infty}(T)$ due to static $v$-changes takes over. The latter appears higher than that of the sputtered layer but still lower than that of bulk v-SiO${ }_{2}$. Reduced $T$-coefficients for $v(T)$ have also

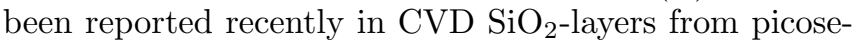
cond acoustics. ${ }^{62}$

Finally one notes that an enhanced TAR-defects density is found in the sputtered layer while, in contrast, the slope of the static $v_{\infty}(T)$-changes is almost reduced by half. This provides further evidence that both anomalies likely have different origins. It was indeed, already observed that the anomalous $T$-induced stiffening of $\mathrm{SiO}_{2}$ network keeps unchanged in permanently densified silica, while TAR defects are fully removed. ${ }^{46}$

\section{SUMMARY AND OUTLOOKS}

To summarize, the acoustical properties of an amorphous $\mathrm{SiO}_{2}$ layer have been investigated by broadband ultrafast acoustic experiment for variable temperature between $20 \mathrm{~K}$ to $300 \mathrm{~K}$. A pulse-echo scheme is used, where a strain pulse is detected in an Al transducer after propagating back and forth through the $\mathrm{SiO}_{2}$ layer. The attenuation of the acoustic pulse within the $\mathrm{SiO}_{2}$ layer is evaluated at different temperature with the help of the effective attenuation law $\alpha(\omega, T)=\alpha_{0}(T) \omega^{p(T)}$ by fitting the successive echoes all at once. Such a law is inferred from the well established phenomenological models describing acoustic attenuation in $\mathrm{v}-\mathrm{SiO}_{2}$ at lower frequencies: coupling to thermally activated relaxation motions of structural defects and interaction with thermal vibrations.

A quantitative agreement is found between the values of $\alpha_{0}(T)$ and $p(T)$ identified in this work and a prediction combining TAR and anharmonicity mechanisms following Ref. 12. The exponent is found to be $p \simeq 1$ near $50 \mathrm{~K}$ while it goes up to $p \leq 2$ at room temperature emphasizing that TAR dominates the damping at low temperature while anharmonicity takes over at sufficiently high temperature. The acoustic absorption from TAR mechanisms in the $\mathrm{SiO}_{2}$ layer is also found to be about one and a half larger than that in bulk v- $\mathrm{SiO}_{2}$. A reasonable explanation would be that the rather cold deposition process used for preparation could generate a larger-than-usual quantity of relaxing defects, and thereby a higher TAR contribution, despite all our efforts to find the optimal growth conditions to sputter $\mathrm{SiO}_{2}$-layers whose physical properties are as close as possible to those of bulk v- $\mathrm{SiO}_{2}$. To our knowledge, only a $\mathrm{SiO}_{2}$ film produced by thermal oxidation of $\mathrm{Si}$ at $1300 \mathrm{~K}$ (i.e. within the glass transition interval) has been found to exhibit internal friction identical to that in bulk v-SiO $2 \cdot{ }^{63}$

Comparing this work to previous ps acoustics measurements, one notes that we have produced the first broadband measurement compatible with the available narrow-band ones (i.e. Refs. 26 and 27). One also notes that the $T$ dependence of acoustics properties in $\mathrm{SiO}_{2}$ layers could be extracted in this work for the first time 
in contrast with the seminal work by Zhu et al. ${ }^{28}$ involving a broadband technique as well. Even if the susceptibility of the TAR-defects density to layer preparation might explain in part these discrepancies, it seems that the fitting procedure including an effective local law for $\alpha(\omega)$ has been crucial in retrieving accurate attenuation coefficients. It is noteworthy that our new results allow raising such issues. Ultrafast acoustics might provide potential for evaluating the TAR-defects concentration in amorphous layers, possibly opening the way to understand how to produce $\mathrm{SiO}_{2}$ layers with low mechanical losses.

An immediate outlook of our work would be to finally search for the onset of the predicted $\omega^{4}$ law over anharmonicity by moving to higher frequencies. Thinner metallic film could provide measurement up to $500 \mathrm{GHz}$. Other setups based on piezo-electric quantum wells ${ }^{29}$ may reach the $\mathrm{THz}$ range close to the boson peak in $\mathrm{v}-\mathrm{SiO}_{2}$. Such experiments might provide the opportunity of directly probing the onset of the expected strong damping regime induced by disorder over anharmonicity on cooling. As the latter mechanism should be $T$ independent, the onset is expected to move down to lower frequency range as $T$ is decreased reducing the anharmonicity damping.

\section{ACKNOWLEDGMENTS}

We thank Loï Becerra, from the Institut des Nanosciences de Paris at UPMC, for the preparation of the thermally grown $\mathrm{SiO}_{2}$ layer sample. This work has been supported by the Agence Nationale de la Recherche (ANR) (Grant No. ANR-11-BS04-008-01, GlassPhon).
* Corresponding author : agnes.huynh@insp.jussieu.fr

1 S. Matsuda, M. Miura, T. Matsuda, M. Ueda, Y. Satoh, and K. y. Hashimoto, IEEE Transactions on Ultrasonics, Ferroelectrics, and Frequency Control 60, 993 (2013).

2 P. R. Saulson, Phys. Rev. D 42, 2437 (1990).

3 K. Numata, M. Ando, K. Yamamoto, S. Otsuka, and K. Tsubono, Phys. Rev. Lett. 91, 260602 (2003).

4 M. Granata, E. Saracco, N. Morgado, A. Cajgfinger, G. Cagnoli, J. Degallaix, V. Dolique, D. Forest, J. Franc, C. Michel, L. Pinard, and R. Flaminio, Phys. Rev. D 93, 012007 (2016).

5 O. L. Anderson and H. E. Bömmel, Journal of the American Ceramic Society 38, 125 (1955).

6 J. Jäckle, L. Piché, W. Arnold, and S. Hunklinger, Journal of Non-Crystalline Solids 20, 365 (1976).

7 K. S. Gilroy and W. A. Phillips, Philos. Mag. 43, 735 (1981).

8 F. Travasso, P. Amico, L. Bosi, F. Cottone, A. Dari, L. Gammaitoni, H. Vocca, and F. Marchesoni, Europhys. Lett. 80, 50008 (2007).

9 J. Fabian and P. B. Allen, Phys. Rev. Lett. 82, 1478 (1999).

10 J.-Y. Duquesne and B. Perrin, Phys. Rev. B 68, 134205 (2003).

11 H. J. Maris (Academic Press, New York, 1971) pp. 279345 .

12 R. Vacher, E. Courtens, and M. Foret, Phys. Rev. B 72, 214205 (2005).

13 J. Jäckle, Z. Phys. 257, 212 (1972).

14 L. Piché, R. Maynard, S. Hunklinger, and J. Jäckle, Phys. Rev. Lett. 32, 1426 (1974).

15 R. C. Zeller and R. O. Pohl, Phys. Rev. B 4, 2029 (1971).

16 B. Rufflé, M. Foret, E. Courtens, R. Vacher, and G. Monaco, Phys. Rev. Lett. 90, 095502 (2003).

17 B. Rufflé, G. Guimbretière, E. Courtens, R. Vacher, and G. Monaco, Phys. Rev. Lett. 96, 045502 (2006).

${ }^{18}$ G. Monaco and V. M. Giordano, Proceedings of the National Academy of Sciences 106, 3659 (2009), http://www.pnas.org/content/106/10/3659.full.pdf.

19 G. Baldi, V. M. Giordano, G. Monaco, and B. Ruta, Phys. Rev. Lett. 104, 195501 (2010).
20 C. Masciovecchio, G. Ruocco, F. Sette, P. Benassi, A. Cunsolo, M. Krisch, V. Mazzacurati, A. Mermet, G. Monaco, and R. Verbeni, Phys. Rev. B 55, 8049 (1997).

21 F. Sette, M. H. Krisch, C. Masciovecchio, G. Ruocco, and G. Monaco, Science 280, 1550 (1998).

22 R. Vacher, S. Ayrinhac, M. Foret, B. Rufflé, and E. Courtens, Phys. Rev. B 74, 012203 (2006).

23 C. Masciovecchio, A. Gessini, S. Di Fonzo, L. Comez, S. C. Santucci, and D. Fioretto, Phys. Rev. Lett. 92, 247401 (2004).

24 P. Benassi, S. Caponi, R. Eramo, A. Fontana, A. Giugni, M. Nardone, M. Sampoli, and G. Viliani, Phys. Rev. B 71, 172201 (2005).

25 B. Rufflé, E. Courtens, and M. Foret, Phys. Rev. B 84, 132201 (2011).

26 S. Ayrinhac, M. Foret, A. Devos, B. Rufflé, E. Courtens, and R. Vacher, Phys. Rev. B 83, 014204 (2011).

27 C. Klieber, E. Peronne, K. Katayama, J. Choi, M. Yamaguchi, T. Pezeril, and K. a. Nelson, Applied Physics Letters 98, 211908 (2011).

28 T. C. Zhu, H. J. Maris, and J. Tauc, Phys. Rev. B 44, 4281 (1991).

29 Y.-C. Wen, S.-H. Guol, H.-P. Chen, J.-K. Sheu, and C.-K. Sun, Applied Physics Letters 99, 051913 (2011).

30 B. Rufflé, D. A. Parshin, E. Courtens, and R. Vacher, Phys. Rev. Lett. 100, 015501 (2008).

31 A. Devos, M. Foret, S. Ayrinhac, P. Emery, and B. Rufflé, Phys. Rev. B 77, 100201 (2008).

32 H. Ogi, T. Shagawa, N. Nakamura, M. Hirao, H. Odaka, and N. Kihara, Phys. Rev. B 78, 134204 (2008).

33 P.-A. Mante, Y.-R. Huang, S.-C. Yang, T.-M. Liu, A. A. Maznev, J.-K. Sheu, and C.-K. Sun, Ultrasonics 56, 52 (2015).

34 E. S. K. Young, A. V. Akimov, R. P. Campion, A. J. Kent, and V. Gusev, Phys. Rev. B 86, 155207 (2012).

35 A. Barranco, F. Yubero, J. Cotrino, J. Espins, J. Ben??tez, T. Rojas, J. Allain, T. Girardeau, J. Rivire, and A. Gonzlez-Elipe, Thin Solid Films 396, 9 (2001).

36 J. Olivares, E. Wegmann, J. Capilla, E. Iborra, M. Clement, L. Vergara, and R. Aigner, IEEE Transactions on 
Ultrasonics, Ferroelectrics and Frequency Control 57, 23 (2010).

37 G. Tas and H. J. Maris, Phys. Rev. B 49, 15046 (1994).

38 O. B. Wright and V. Gusev, IEEE Transactions on Ultrasonics, Ferroelectrics and Frequency Control 42, 331 (1995).

39 B. Perrin, B. Bonello, J. C. Jeannet, and E. Romatet, Prog. Nat. Sci. 6, 444 (1996).

40 B. Perrin, B. Bonello, J.-C. Jeannet, and E. Romatet, Physica B 219-220, 681 (1996).

41 See Supplemental Material at [URL will be inserted by publisher].

42 S. Ayrinhac, B. Rufflé, M. Foret, H. Tran, S. Clément, R. Vialla, R. Vacher, J. C. Chervin, P. Munsch, and A. Polian, Phys. Rev. B 84, 024201 (2011).

43 W. A. Phillips, Rep. Prog. Phys. 50, 1657 (1987).

44 D. Tielbürger, R. Merz, R. Ehrenfels, and S. Hunklinger, Phys. Rev. B 45, 2750 (1992).

${ }^{45}$ R. Keil, G. Kasper, and S. Hunklinger, J. Non-Cryst. Solids 164-166, 1183 (1993).

${ }^{46}$ E. Rat, M. Foret, G. Massiera, R. Vialla, M. Arai, R. Vacher, and E. Courtens, Phys. Rev. B 72, 214204 (2005).

47 V. Gurevich, Transport in phonon systems, edited by V. Agranovich and A. Maradudin, Modern problems in condensed matter sciences, Vol. 18 (North-Holland, Amsterdam Oxford New York Tokyo, 1986).

48 S. Hunklinger and M. v.Schickfus, "Acoustic and dielectric properties of glasses at low temperatures," (SpringerVerlag, Berlin, 1981) Chap. 6, pp. 79-105.

49 R. E. Strakna, Phys. Rev. 123, 2020 (1961).

${ }^{50}$ K. Döring, S. Rau, G. Weiss, J. Arndt, and S. Hunklinger, Physics Letters A 184, 464 (1994).
51 G. Weiss, A. Daum, M. Sohn, and J. Arndt, Physica B 219-220, 290 (1996).

52 C. Weigel, M. Foret, B. Hehlen, M. Kint, S. Clément, A. Polian, R. Vacher, and B. Rufflé, Phys. Rev. B 93, 224303 (2016).

53 R. J. Hemley, H. K. Mao, P. M. Bell, and B. O. Mysen, Phys. Rev. Lett. 57, 747 (1986).

54 R. Hamdan, J. P. Trinastic, and H. P. Cheng, The Journal of Chemical Physics 141, 054501 (2014), http://dx.doi.org/10.1063/1.4890958.

55 C. R. Billman, J. P. Trinastic, D. J. Davis, R. Hamdan, and H.-P. Cheng, Phys. Rev. B 95, 014109 (2017).

56 J. T. Krause and C. R. Kurkjian, Journal of the American Ceramic Society 51, 226 (1968).

57 R. Youngman, J. Kieffer, J. Bass, and L. Duffrene, Journal of Non-Crystalline Solids 222, 190 (1997).

58 A. Polian, D. Vo-Thanh, and P. Richet, EPL (Europhysics Letters) 57, 375 (2002).

59 A. G. Kalampounias, S. N. Yannopoulos, and G. N. Papatheodorou, The Journal of Chemical Physics 124, 014504 (2006), http://dx.doi.org/10.1063/1.2136878.

${ }^{60}$ L. Huang and J. Kieffer, Phys. Rev. B 69, 224203 (2004).

61 Y. Liang, C. R. Miranda, and S. Scandolo, Phys. Rev. B 75, 024205 (2007).

62 A. Nagakubo, H. Ogi, H. Ishida, M. Hirao, T. Yokoyama, and T. Nishihara, Journal of Applied Physics 118, 014307 (2015), http://dx.doi.org/10.1063/1.4923353.

63 B. E. White and R. O. Pohl, Phys. Rev. Lett. 75, 4437 (1995). 